\title{
ChemComm
}

\section{High internal phase emulsion (HIPE) organogels prepared from charge-driven assembled polymer organogels $\dagger$}

\author{
Cite this: Chem. Commun., 2013 \\ 49, 11803 \\ Received 23rd September 2013, \\ Accepted 24th October 2013
}

DOI: $10.1039 /$ c3cc47263a

\author{
Tao Zhang and Qipeng Guo*
}

www.rsc.org/chemcomm

\begin{abstract}
We prepared HIPE organogels from polymer organogels which were pre-formed based on a triblock ionomer and polypropylenimine dendrimers via charge-induced assembly. The morphologies of the HIPE organogels are little changed with salt concentrations while the mechanical strength of the organogels can be tuned by PPI dendrimers.
\end{abstract}

High internal phase emulsions (HIPEs) are two-phase systems with the total fraction of the dispersed/internal phase being over $74 \%$ by volume. ${ }^{1}$ HIPEs are usually highly viscous due to the low fraction of the continuous phase, and they are also called gel emulsions. ${ }^{2}$ HIPEs are widely used in foods, ${ }^{3}$ chemical industry, ${ }^{4}$ medicine ${ }^{5}$ and in preparation of materials having porous structures or low density. ${ }^{6}$ Because of the fascinating properties and widespread applications, development of a new route to prepare HIPEs has attracted considerable attention.

HIPEs are usually stabilized by surfactants and particles. As stabilizers, surfactants are commonly used despite their low efficiency. Indeed, $5-50 \%(\mathrm{w} / \mathrm{v})$ of the volume of the continuous phase is needed to form HIPEs. ${ }^{7}$ Solid particles of a few nanometres to micrometres have also been used to stabilize HIPEs, which are known as Pickering emulsions. ${ }^{8}$ However, phase inversion may occur in HIPEs stabilized by particles, although a variety of inorganic particles functioning as stabilizers have been reported from silica, titanium oxide to clay.

Recently, organic gels have attracted considerable attention as stabilizers to form HIPEs. ${ }^{9}$ Ngai and co-workers reported the preparation of HIPE organogels and hydrogels from poly(methacrylic acid)-based microgel particles, representing a kind of physically crosslinked gel without flow behaviour. ${ }^{10,11}$ HIPE organogels and hydrogels were also prepared from microgels which formed via hydrogen bonding interaction by incorporating functional groups onto polymer chains. ${ }^{12}$ Fang and co-workers

Polymers Research Group, Institute for Frontier Materials, Deakin University, Locked Bag 2000, Geelong, Victoria 3220, Australia.E-mail: qguo@deakin.edu.au; Fax: +61 35227 1103; Tel: +6135227 2802

$\dagger$ Electronic supplementary information (ESI) available: Preparation of organogels and HIPE organogels, ionic interaction between SSEBS and PPI dendrimers, responsiveness of organogels and movies. See DOI: 10.1039/c3cc47263a reported preparation of HIPE organogels from bulk organogels based on low molecular weight gelators and found that the mechanical strength of the HIPE organogels was dominated by the continuous phase. ${ }^{6,14}$

Herein we demonstrate for the first time that HIPE organogels can be prepared from polymer organogels which are usually inexpensive in comparison with organogels prepared from low molecular weight organogelators. ${ }^{13}$ In our previous study, we developed a new route to prepare multiresponsive organogels by charge-induced assembly of a triblock ionomer, sulfonated polystyrene-block-poly(ethylene-ran-butylene)-block-polystyrene (SSEBS), and a diblock copolymer polystyrene-block-poly(2-vinyl pyridine) (PS- $b$-P2VP). ${ }^{14}$

By tuning the hydrophilicity of amines used, ionic complexes can stabilize water droplets in organic solvents, leading to the macrophase separation in the gels. When the volume fraction of water droplets in gels exceeds 74\%, HIPE gels form. In this work, the pre-formed organogels were based on triblock ionomer SSEBS and polypropylenimine (PPI) dendrimers. PPI dendrimers of generation 1 (DAB-4), generation 2 (DAB-8) and generation 3 (DAB-16) were used (see ESI 1†). The organogels were prepared by mixing SSEBS solution and PPI dendrimer solutions with an equimolar amount of sulfonic acid groups and amine moieties, the organogels were formed in 20-30 seconds upon mixing as confirmed by the tube-inversion method (ESI, $\uparrow$ Movie (a): organogel formation). ${ }^{15}$ All the organogels were slightly yellowish without visible heterogeneity, indicating that no macrophase separation occurred in the organogels. As expected, the formation of gels was induced by ionic interaction between SSEBS and PPI dendrimers, which was verified by FTIR study (see ESI $2 \dagger$ ).

The formation of organogels was further confirmed by rheological measurement, and the results for dynamic frequency measurements are shown in Fig. 1. For all organogels, the elastic moduli $\left(G^{\prime}\right)$ are higher than the corresponding viscous moduli $\left(G^{\prime \prime}\right)$ at all frequencies ranging from 0.5 to $100 \mathrm{rad} \mathrm{s}^{-1}$, demonstrating the rubber-like behaviour in the range. It can be seen that both the elastic and the viscous moduli increase with the molecular weight of PPI dendrimers, indicating that the mechanical strength of the organogels can be tuned by PPI dendrimers. 


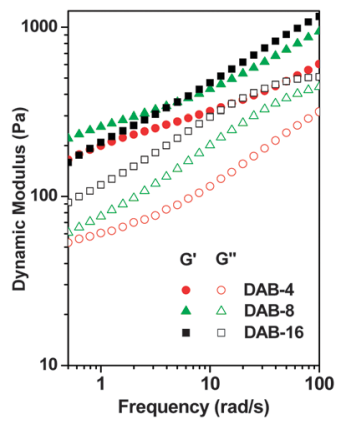

Fig. 1 Elastic moduli $G^{\prime}$ and viscous moduli $G^{\prime \prime}$ of SSEBS/DAB-4, SSEBS/DAB-8 and SSEBS/DAB-16 organogels as a function of oscillatory shear frequency.

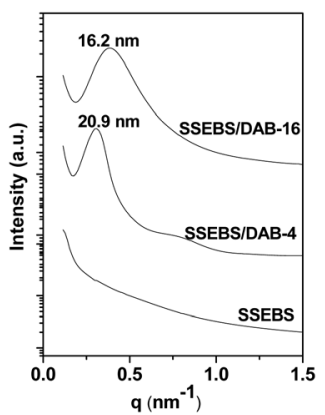

Fig. 2 SAXS profiles of SSEBS solution, SSEBS/DAB-4 and SSEBS/DAB-16 gels.

The morphologies of the organogels were investigated by smallangle X-ray scattering (SAXS) at room temperature, and the SAXS profiles are shown in Fig. 2. Although there is no obvious peak observed in the figure for SSEBS solution, well-defined peaks are noted for SSEBS/DAB-4 and SSEBS/DAB-16 organogels, demonstrating that microphase separation occurred in the gels. Since the first-order peak is broad and the resolution of the higher order peak becomes poorer with the increase of ionic interaction for oil-swelling SSEBS gels, ${ }^{16}$ it is impossible to clarify the exact morphology of the organogels. According to the composition of the organogels and similar results reported ${ }^{14,16,17}$ it is believed that the ionic microdomains were stabilized by solvophilic blocks of SSEBS in the gels. Based on the $q$ values, the average distances between neighbouring domains were calculated to be 20.9 and $16.2 \mathrm{~nm}$ for SSEBS/DAB-4 and SSEBS/DAB-16 organogels, respectively. The peak for SSEBS/DAB-4 organogels is much narrower than that for SSEBS/DAB-16 organogels, indicating the existence of more ordered structures in the SSEBS/DAB-4 organogels.

To confirm that the organogels were stabilized by the presence of ionic microdomains, acetic acid, triethylamine and their salts were added to the pre-formed organogels, as they could affect the strength of ionic interaction. In our experiments, when $2 \%(\mathrm{v} / \mathrm{v})$ of acetic acid, triethylamine and their salts were added to the preformed organogels, the gels transformed into a liquid, indicating the breakdown of networks. A quantitative study was carried out using dynamic frequency measurements, showing that the preformed organogels are responsive to acids, amines or salts even when only $0.5 \%(\mathrm{v} / \mathrm{v})$ is added (see ESI $3 \dagger)$. These results revealed that the mechanism for the formation of SSEBS/PPI dendrimer organogels is similar to that of SSEBS/PS- $b$-P2VP organogels. ${ }^{14}$
The ionic complexes form charged microdomains, and the domains are connected and stabilized by the middle blocks of SSEBS to form gels.

The ionic complexes are hydrophilic, and the charged domains in the complexes should be able to dissolve or partially dissolve in water. However, upon addition of water to the pre-formed organogels, a precipitate formed. In order to avoid precipitation, tetrahydrofuran (THF) was added. When a water-THF mixture $(4: 1, \mathrm{v} / \mathrm{v})$ was added to the pre-formed organogels, new white gels formed and the gel formation was confirmed by the tube inversion method (ESI, $\uparrow$ Movie (b): HIPE organogel formation) and rheological measurement. The fraction of the water phase could be as high as $80 \%$, which was studied by control experiments with the same solvents. Although white gels can be obtained by simple blending of SSEBS/DAB-4 organogels with a water-THF mixture, it is impossible to form gels from SSEBS/ DAB-8 and SSEBS/DAB-16 organogels without addition of $\mathrm{NaCl}$. Our experiments showed that the HIPE organogels can also be obtained by adding other salts such as $\mathrm{KCl}$ or $\mathrm{Na}_{2} \mathrm{SO}_{4}$. From the results, it is reasonable to believe that an electrolyte plays an important role in the formation of the gels.

To study the structure of newly formed gels, conductivity of the gels was measured. The results showed that the conductivity of newly formed white gels was $0 \mu \mathrm{S} \mathrm{cm}^{-1}$, so the gels had a structure of water droplets dispersed in the organic continuous phase according to results obtained from control experiments, which showed that the conductivities of the water phase and the organic phase were 102.4 and $0 \mu \mathrm{S} \mathrm{cm}^{-1}$, respectively. As the volume fraction of the internal phase was over $74 \%$, high internal phase organogels formed.

The structure of the high internal phase organogel was further investigated by confocal microscopy. The confocal image of the gel in Fig. 3 confirmed the formation of water-in-oil organogels, with toluene (pyrene labelled) as the continuous organogel phase with water droplets entrapped. The size of water droplets varied from several to tens of micrometres, which was in the size range of emulsions, and thus HIPE organogels formed.

The stability of the HIPE organogels was examined in closed vials. About $5 \%(\mathrm{w} / \mathrm{v})$ of water was separated from the HIPE organogels in the first two weeks, and no further change was observed in the following seven months.

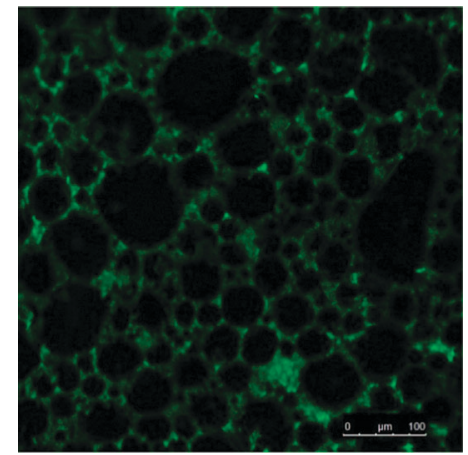

Fig. 3 Confocal image of HIPE gels prepared from SSEBS/DAB-4 with $0.25 \mathrm{M}$ $\mathrm{NaCl}$ added, excited by laser with a wavelength of $405 \mathrm{~nm}$ for the pyrene labelled organic phase, scale bar: $100 \mu \mathrm{m}$. 


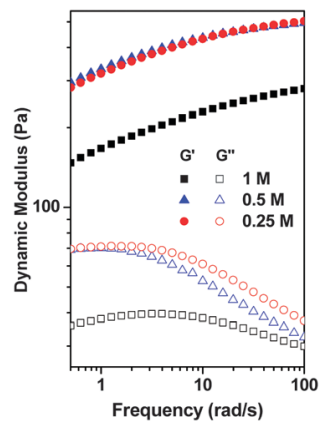

Fig. 4 Dynamic moduli $G^{\prime}$ and $G^{\prime \prime}$ of HIPE organogels prepared from SSEBS/DAB-4 with different $\mathrm{NaCl}$ concentrations as a function of oscillatory shear frequency.
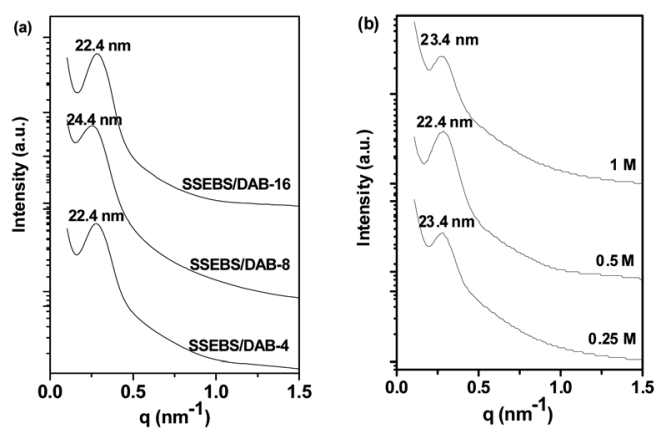

Fig. 5 SAXS profiles of HIPE organogels (a) with SSEBS/DAB-4, SSEBS/DAB-8 and SSEBS/DAB-16 with $0.5 \mathrm{M} \mathrm{NaCl}$, (b) SSEBS/DAB-4 with $0.25,0.5$ and $1 \mathrm{M} \mathrm{NaCl}$.

The effect of salt concentration on mechanical properties of HIPE organogels was studied by rheological measurement. Fig. 4 presents SSEBS/DAB-4 HIPE organogels with different $\mathrm{NaCl}$ concentrations, and it is observed that $G^{\prime}$ always exceed $G^{\prime \prime}$ at all frequencies ranging from 0.5 to $100 \mathrm{rad} \mathrm{s}^{-1}$, indicating that HIPE organogels are formed. Both $G^{\prime}$ and $G^{\prime \prime}$ of HIPE organogels decrease with the increase of salt concentration. To our knowledge, this result is quite different from that reported for conventional HIPE organogels, where high concentration of the electrolyte leads to the formation of strong HIPE organogels. ${ }^{10}$ This unusual result could result from the salt screen effect. ${ }^{18}$ With the increase of salt concentrations in the water phase, the electrostatic interaction between $-\mathrm{SO}_{3} \mathrm{H}$ groups on SSEBS and amine moieties on PPI decreases, resulting in the decrease in the modulus of HIPE gels.

To reveal the morphology of HIPE organogels, SAXS experiments were carried out at room temperature and the results are shown in Fig. 5. In SSEBS organogels, the relaxation behaviour of charged domains controls the morphology of gels. ${ }^{16}$ After addition of water to the pre-formed SSEBS organogels, water would dissolve or swell ionic domains, which decreases the relaxation time and increases the mobility of charged blocks. As a result, the morphology of HIPE organogels would change. From Fig. 5(a), it can be seen that well-defined peaks occur for all the HIPE organogels, demonstrating the persistence of microphase separation. The average distances between neighbouring domains of the HIPE organogels (Fig. 5(a)) are considerably increased compared to the pre-formed organogels (Fig. 2). As the ionic domains are hydrophilic after addition of water, the interaction between endblocks on triblock ionomers decreases and the balance between endblocks and midblocks determines the phase separation, leading to the nearly unchanged morphology of all the HIPE organogels. The SAXS profiles in Fig. 5(b) demonstrate that the morphologies of HIPE organogels are little affected by different salt concentrations, indicating that salt concentrations do not dominate the microphase separation in HIPE organogels though they affect the formation and mechanical strength of HIPE organogels.

In conclusion, we have presented the first example of HIPE organogels prepared from polymer organogels. The polymer organogels are formed from a triblock ionomer SSEBS and PPI dendrimers by charge-induced assembly. Upon addition of a water-THF mixture into the organogels, the charged domains in the pre-formed organogels stabilize water droplets, resulting in the transition from organogels to HIPE organogels. The morphologies of HIPE organogels are little changed with the salt concentrations though the salt concentrations affect the formation and mechanical strength of HIPE organogels. The obtained HIPE organogels should have potential for applications such as preparation of porous materials and separation membranes.

SAXS measurements were conducted on the SAXS beam-line at the Australian Synchrotron, Victoria, Australia, and we would like to thank Dr Patrick G. Hartley for sharing his Foundation Investor Beamtime with us. The authors appreciate Zhifeng Yi of Deakin University for his assistance in using the confocal microscope.

\section{Notes and references}

1 K. J. Lissant, B. W. Peace, S. H. Wu and K. G. Mayhan, J. Colloid Interface Sci., 1974, 47, 416.

2 A. Menner, R. Powell and A. Bismarck, Macromolecules, 2006, 39, 2034.

3 V. L. Sok Line, G. E. Remondetto and M. Subirade, Food Hydrocolloids, 2005, 19, 269.

4 A. Imhof and D. J. Pine, Nature, 1997, 389, 948.

5 S. Rocca, S. Muller and M. J. Stébé, J. Controlled Release, 1999, 61, 251.

6 X. Chen, K. Liu, P. He, H. Zhang and Y. Fang, Langmuir, 2012, 28, 9275; P. J. Colver and S. A. F. Bon, Chem. Mater., 2007, 19, 1537.

7 J. M. Williams, Langmuir, 1991, 7, 1370; A. Barbetta and N. R. Cameron, Macromolecules, 2004, 37, 3188.

8 V. O. Ikem, A. Menner and A. Bismarck, Angew. Chem., Int. Ed., 2008, 47, 8277; A. Menner, V. Ikem, M. Salgueiro, M. S. P. Shaffer and A. Bismarck, Chem. Commun., 2007, 4274.

9 S. Zhang and J. Chen, Chem. Commun., 2009, 2217.

10 G. Sun, Z. Li and T. Ngai, Angew. Chem., Int. Ed., 2010, 49, 2163.

11 Z. Li, T. Ming, J. Wang and T. Ngai, Angew. Chem., Int. Ed., 2009, 48, 8490; Z. Li and T. Ngai, Langmuir, 2010, 26, 5088.

12 Y. Chen, N. Ballard, F. Gayet and S. A. F. Bon, Chem. Commun., 2012, 48, 1117; Y. Chen, N. Ballard and S. A. F. Bon, Chem. Commun., 2013, 49, 1524.

13 M. Suzuki and K. Hanabusa, Chem. Soc. Rev., 2010, 39, 455.

14 T. Zhang and Q. Guo, Chem. Commun., 2013, 49, 5076.

15 A. R. Hirst and D. K. Smith, Langmuir, 2004, 20, 10851.

16 X. Lu, W. P. Steckle Jr. and R. A. Weiss, Macromolecules, 1993, 26, 6525.

17 X. Lu, W. P. Steckle and R. A. Weiss, Macromolecules, 1993, 26, 5876; S. Wu, S. Peng, N. Hameed, Q. Guo and Y.-W. Mai, Soft Matter, 2012, 8, 688; S. Wu, Q. Guo, S. Peng, N. Hameed, M. Kraska, B. Stühn and Y.-W. Mai, Macromolecules, 2012, 45, 3829; S. Wu, Q. Guo, T. Zhang and Y.-W. Mai, Soft Matter, 2013, 9, 2662.

18 R. Chollakup, W. Smitthipong, C. D. Eisenbach and M. Tirrell, Macromolecules, 2010, 43, 2518; J. N. Hunt, K. E. Feldman, N. A. Lynd, J. Deek, L. M. Campos, J. M. Spruell, B. M. Hernandez, E. J. Kramer and C. J. Hawker, Adv. Mater., 2011, 23, 2327. 